\title{
COVID-19-Induced Stroke and the Potential of Using Mesenchymal Stem Cells-Derived Extracellular Vesicles in the Regulation of Neuroinflammation
}

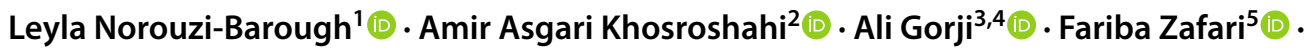 \\ Mohammad Shahverdi Shahraki ${ }^{6}\left[\right.$ Sadegh Shirian ${ }^{6,7}(\mathbb{D}$
}

Received: 17 May 2021 / Accepted: 9 November 2021 / Published online: 13 January 2022

(c) The Author(s), under exclusive licence to Springer Science+Business Media, LLC, part of Springer Nature 2022

\begin{abstract}
Ischemic stroke (IS) is a known neurological complication of COVID-19 infection, which is associated with high mortality and disability. Following IS, secondary neuroinflammation that occurs can play both harmful and beneficial roles and lead to further injury or repair of damaged neuronal tissue, respectively. Since inflammation plays a pivotal role in the pathogenesis of COVID-19-induced stroke, targeting neuroinflammation could be an effective strategy for modulating the immune responses following ischemic events. Numerous investigations have indicated that the application of mesenchymal stem cells-derived extracellular vesicles (MSC-EVs) improves functional recovery following stroke, mainly through reducing neuroinflammation as well as promoting neurogenesis and angiogenesis. Therefore, MSC-EVs can be applied for the regulation of SARS-CoV-2-mediated inflammation and the management of COVID-19- related ischemic events. In this study, we have first described the advantages and disadvantages of neuroinflammation in the pathological evolution after IS and summarized the characteristics of neuroinflammation in COVID-19-related stroke. Then, we have discussed the potential benefit of MSC-EVs in the regulation of inflammatory responses after COVID-19-induced ischemic events.
\end{abstract}

Keywords COVID-19 $\cdot$ Stroke $\cdot$ Neuroinflammation $\cdot$ Mesenchymal stem cells $\cdot$ Extracellular vesicles $\cdot$ Exosome

Sadegh Shirian

shirian@shirazu.ac.ir; shirian85@gmail.com

1 Acquired Immunodeficiency Research Center, Isfahan University of Medical Sciences, Isfahan, Iran

2 Islamic Azad University, Kazerun Branch, Kazerun, Iran

3 Epilepsy Research Center, Department of Neurosurgery, Westfälische Wilhelms-Universitat Münster, Munster, Germany

4 Shefa Neuroscience Research Center, Khatam Alanbia Hospital, Tehran, Iran

5 Cellular and Molecular Research Center, Research Institute for Prevention of Non- Communicable Disease, Qazvin University of Medical Sciences, Qazvin, Iran

6 Department of Pathology, School of Veterinary Medicine, Shahrekord University, Shahrekord, Iran

7 Shiraz Molecular Pathology Research Center, Dr. Daneshbod Pathol Lab, Shiraz, Iran

\section{Introduction}

Stroke is the second leading cause of mortality worldwide and the most common cause of long-term disability in Western countries (Rajkovic et al.2018; Jayaraj et al. 2019). This neurological disorder occurs following the acute injury of the brain by a vascular reason (Campbell and Khatri 2020). Ischemic stroke (IS) is the most common type of stroke due to the reduced blood flow to a certain part of the brain and accounts for about $85 \%$ of all acute strokes (Tadi and Lui 2020). IS leads to energy depletion and triggers excitotoxicity and neuroinflammation in the vicinity of brain injury (Liu et al. 2017).

Several disorders, such as hematologic diseases, cardiovascular abnormalities, and infection increase the risk of IS (Woldu 2020; Ferro and Infante 2021; Virani et al 2021). Coronavirus disease 2019 (COVID-19) results from SARS-CoV-2 is an inflammatory disorder (Leisman et al. 2020; Gorji and Khaleghi Ghadiri 2021), which can affect the central nervous system (CNS) (Soltani Zangbar et al. 2021; Rezaeitalab et al. 2021). COVID-19 predisposes 
some patients to a wide range of neurological complications, including stroke (Ntaios et al. 2020). It is estimated that stroke occurs in about $2-6 \%$ of the hospitalized patients with COVID-19 (Ellul et al. 2020). It has been shown that SARS-CoV-2 is more likely to induce thrombotic complications, such as IS compared to influenza infection (Merkler et al. 2020). Although COVID-19-induced stroke is more common in elderly people with underlying disorders, such as hypertension, atherosclerosis, or atrial fibrillation, it is mainly linked to a hypercoagulable state caused by systemic inflammation and cytokine storm (Spence et al. 2020; Fifi and Mocco 2020). Aside from the neuroinflammation induced by systemic immune activation, direct invasion of SARS-CoV-2 through binding its spike protein to the receptor angiotensin-converting enzyme 2 (ACE2) may lead to neuroinflammation and cytokine release (Sriwastava et al. 2021; Song et al. 2021).

Numerous investigations have indicated that the application of MSC-EVs improves functional recovery following stroke, mainly through reducing neuroinflammation as well as promoting neurogenesis and angiogenesis (Jiang et al. 2018; Deng et al 2019; Yang et al. 2020). This review aimed to summarize the features of neuroinflammation induced by COVID-19 and non-COVID-19 stroke and then discuss therapeutic effects and underlying mechanisms of action of MSCs-EVs in the modulation of neuroinflammation and improvement of functional recovery following stroke.

\section{Neuroinflammation in the Pathological Evolution After IS}

Neuroinflammation is a major and primary pathological event involved in IS (Fig. 1) (Lian et al. 2021). Indeed, neuroinflammation is a common feature in a wide variety of CNS diseases, such as IS (Stephenson et al. 2018). Within the CNS, an inflammatory response is considered as the capability of the neuronal tissues to drive neuroprotection against pathological conditions (Mendiola and Cardona 2018). The initiation and development of neuroinflammation

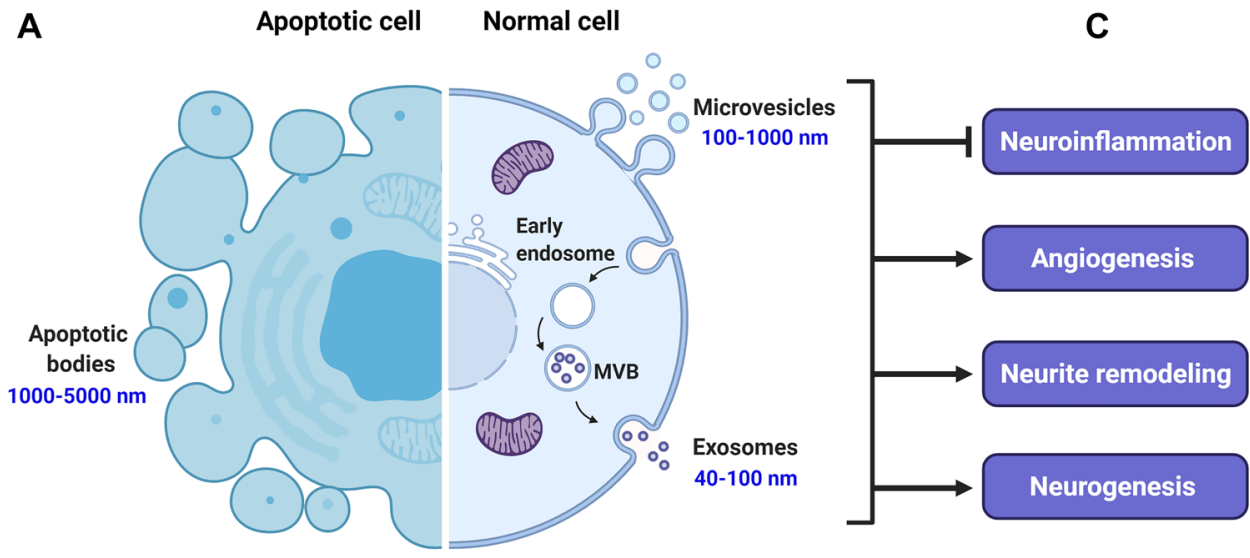

\section{B Extracellular Vesicle Markers \& Contents}

Apoptotoc bodies
Markers
Histones, gp96, protein,
phosphatidylserine

Microvesicles
Markers
Integrins, selectins, CD40
Contents
Nucleic acids (mRNA,
miRNA \& other non-coding
RNAs), proteins

Fig. 1 Schematic overview of the general mechanisms of neuroinflammation after ischemic stroke, Ischemic stroke occurs following a blood vessel occlusion due to a thrombus or embolus. Brain ischemia results in oxygen and ATP depletion and subsequently acute neuronal damage in the infarcted tissue. Then, danger-associated molecular patterns (DAMPs) are released by injured and dying neurons which in turn activate microglia, astrocytes, and endothelial cells, leading to the produce the proinflammatory factors such as IL-1, IL-6 and TNF, reactive oxygen species (ROS), matrix metalloproteinases (MMPs), and vascular endothelial growth factor (VEGF). These molecules trigger BBB dysfunction by endothelial downregulation of tight junction proteins and upregulation of cell adhesion molecules including vascular cell adhesion molecules (VCAM) and intracellular adhesions molecule-1 (ICAM-1), leading to migration and infiltration of circulating leukocytes, mainly neutrophils through the disrupted BBB into the ischemia area. Activated neutrophils release proinflammatory mediators which cause further activation of microglia and astrocytes. This pathological cascade leads to neuronal cell death, edema, and hemorrhage, which in turn cause further neuroinflammation and tissue damage 
presumably are based on the interaction between glial cells, neurons, and immune cells (Skaper et al. 2017). Glial cells, in particular microglia, present a central role in the initiation of neuroinflammation via producing proinflammatory mediators (Almeida et al. 2020). In homeostatic conditions, neurons actively induce anti-inflammatory signals and subsequently prevent microglia polarization to the inflammatory M1 phenotype (Bernaus et al. 2020). In response to various stresses such as IS, microglial cells are rapidly activated and polarized into the proinflammatory M1 or the anti-inflammatory M2 phenotypes, which are involved in secondary brain damage or repair, respectively (Jiang et al. 2020).

Microglia activation is the hallmark of neuroinflammation and occurs immediately following stroke (He et al. 2020). The M1 polarized microglia are functionally characterized by the capability of eliminating microorganisms or tumor cells (Lanza et al. 2021). They also release proinflammatory cytokines (IL-1 $\beta$, IL-6, IL-12, IFN- $\gamma$, and TNF), chemokines (CCL-2, CCL-20), CXCL-10, and cytotoxic substances, including reactive oxygen species (ROS), reactive nitrogen species, excitatory amino acids, and prostaglandins E2 (Liu et al. 2019). These proinflammatory factors, particularly inducible nitric oxide synthase, have cytotoxic effects on neurons, leading to neuronal loss, disruption of the blood-brain barrier (BBB) and the degradation of the extracellular matrix (ECM) (Zhang et al. 2021a, b). Alternatively, M2 microglia induces phagocytosis of dead neurons stronger and is associated with neural survival, reduction of brain damage, and restriction of destructive immune response (Lanza et al. 2021; Zhang et al. 2021a, b). M2 phenotype is activated to exert a beneficial role following IS by producing anti-inflammatory and neuroprotective mediators including, IL-10, IL-4, and TGF- $\beta$ (Jiang et al. 2020). Among these, IL-4 is an important cytokine involved in the improvement of functional recovery after IS (Zheng and Wong Division 2019).

The function and nature of neuroinflammation can be either supportive or destructive based on the conditions and the intensity as well as the duration of inflammation (Liu et al. 2017). Evidence indicates that the proinflammatory state is probably dominant in the acute phase of IS (Jiang et al. 2020). Although the production of transient proinflammatory mediators, particularly cytokines, in the early and acute stages of brain injuries plays a neuroprotective role, including promoting injury recovery and axonal re-growth (Liu et al. 2017), long-term and supra-physiological production of inflammatory mediators following uncontrolled and persistent glial activation causes chronic inflammation and leads to secondary damage (Bronzuoli et al. 2016). The pro-inflammatory mediators are detrimental to the hypoxic tissues. Glial cells can damage healthy neurons and lead to decreased neuronal plasticity and cognitive impairments (Liu et al. 2017;
Jurga et al. 2020). Therefore, the imbalance between M1 and M2 polarization states and shifting to the M1 phenotype contributes to the pathogenesis of neuroinflammation following brain injury events such as IS (Liu et al. 2019). Thus, modulation of the microglial phenotype is one of the most important strategies to reduce inflammatory responses and promotion of brain repair, via reducing brain edema, improving the integrity of white matter, promoting neurogenesis, and recovering motor function (Liu et al. 2019; Jiang et al. 2020). However, the sustained inhibition of microglia at the early stages of stroke interferes with neurogenesis (Xiong et al. 2016). Therefore, a desirable anti-inflammatory agent for alleviating IS should maintain the physiological activation of glial cells (Bronzuoli et al. 2016).

Both in vitro and in vivo studies have highlighted the crosstalk of microglia with astrocytes in the neurovascular unit (Bernaus et al. 2020). These studies have indicated that microglia are more sensitive to damage and activated in the acute phase of IS. Then, the activated microglia produce signaling molecules, such as TNF, IL-1, and IL-1 receptor antagonist (IL-1Ra) and trigger reactive astrocytes (Liu et al. 2020a, b). Astrocytes use the same neuroprotective and immunoregulatory mechanisms for both the maintenance of homeostasis and response to injury (Cekanaviciute and Buckwalter 2016). Similar to microglia, astrocytes release a variety of pro and anti-inflammatory cytokines and chemokines under different conditions (Whitney et al. 2009). Astrocytes are known as the important in situ regulators of the neuroinflammatory processes in stroke and gain the reactive phenotype in the subacute time frame of 2 days to 1 week after stroke, which is associated with the expression of cytokines and peak inflammatory response (Cekanaviciute and Buckwalter 2016).

The reactive astrocytes regulate immune responses by infiltration and activation of immune cells, which motivate macrophages to acquire either proinflammatory or antiinflammatory phenotypes. They exert both harmful (A1 reactive astrocytes) and beneficial (A2 reactive astrocytes) effects on neuroinflammation and neurological outcomes following brain injury (Cekanaviciute and Buckwalter 2016). Evidence indicated that the kind of stimuli during neuroinflammation may switch the function of astrocytes from beneficial to detrimental (Colombo and Farina 2016). The prolonged expression of proinflammatory mediators, such as TNF, IL-12, IL-17, CXCL10, intensify the infiltration of T lymphocytes and myeloid cells into the CNS and may result in uncontrolled chronic inflammation (Cekanaviciute and Buckwalter 2016). It may be helpful to moderately weaken the activation and shorten the M1/A1 or strengthen the A2/M2 timeline for better brain repair and functional recovery after IS (Liu et al 2020a, b). 


\section{IS and Neuroinflammation in COVID-19 Patients}

Ischemic stroke is known as a presenting feature of COVID-19 and is associated with a poor prognosis and high mortality in patients with COVID-19 (Shah et al. 2020; Zhang et al. 2021a, b). Although the stroke in patients with COVID-19 could be due to aging and underlying comorbidities, such as hypertension, diabetes, malignancy, and cardiovascular disorders, COVID-19-induced stroke is mainly linked to a hypercoagulable state caused by systemic inflammation and is termed sepsis-induced coagulopathy (Spence et al. 2020; Fif and Mocco 2020; Jarrahi et al. 2020). Indeed, blood coagulation plays a fundamental role in COVID-19-mediated stroke (Yaghi et al. 2020).

In addition to triggering a hypercoagulable state, a direct viral infection of endothelial cells can result in endotheliitis and eventually endothelial dysfunction (Nannoni et al. 2021). Inflammation and apoptosis of endothelium following infection by SARS-CoV-2 have been reported at autopsy samples from lung, heart, kidney, and bowel (Pezzini and Padovani 2020). Similar to these organs, SARS-CoV-2 can directly infect BBB endothelium through binding to ACE-2 receptor and increase the risk of viral-induced vasculitis, BBB injury, and subsequently IS as the long-term outcome for patients (Berger 2020; Bodro et al. 2021). Moreover, infection of endothelial cells can be followed by perivascular astrocytes and macrophages due to proximity with infected endothelium. Subsequently, viral infection may be transmitted to microglia and neurons (Murta et al. 2020). On the other hand, BBB breakdown as an outcome of systemic inflammation and cytokine storm can facilitate SARS-CoV-2 penetration to the CNS. Excessive levels of proinflammatory cytokines, particularly IL-6, TNF, MIP-1 $\alpha$, CXCL10 (IP-10), G-CSF, $\mathrm{C}$-reactive protein, and ferritin can destabilize the integrity of tight junctions between BBB endothelial cells, thus enabling viral entry (Alam et al. 2020). Subsequently, microglia and astrocytes as part of local neuroinflammatory responses, express pattern recognition receptors (PRRs), to identify and interact with pathogen-associated molecular patterns (PAMP) required initiating and/or augmenting innate immunity within the CNS (Murta et al. 2020).

All these pathways result in astrocytes and microglia infection (as the viral hosts) or their hyperactivation after receiving proinflammatory signals arise from systemic inflammation, which finally leads to shifting to a proinflammatory state and expanding neuroinflammation (Murta et al. 2020). Moreover, evidence has indicated that invasion of SARS-CoV-2 through the olfactory system into the brain parenchyma can drive neuroinflammation (De
Melo et al. 2020). Furthermore, SARS-CoV-2may reach the CNS through other peripheral nerves including the vagus nerve, which enables the virus to access the brain stem from the lungs and gut (the lung-gut-brain axis) (Chigr et al. 2020; Zhang et al. 2020). This may explain a combination of the lung, gastrointestinal, and neurological involvement during infection with COVID-19 in some patients (Alam et al. 2020).Furthermore, there is a less common route for penetration and spreading of the virus through the lymphatic drainage system (Finsterer and Stollberger 2020). Eventually, all these events are associated with the increased risk of ischemic or hemorrhagic stroke and neuroinflammation (Bodro et al. 2021).

\section{The Potential Use of MSC-Derived EVs in Modulating Neuroinflammation Following CoVID-19-Induced Stroke}

Currently, there is no specific and effective antiviral treatment available for COVID-19 patients, and countless clinical investigations are trying to find therapeutic approaches for different forms of the disease. Physicians and researchers are applying and evaluating the effect of mesenchymal stromal/ stem cells (MSCs) or their secretomes, including EVs, in patients with COVID-19. These ongoing studies will provide significant information for the treatment of COVID-19 or the prevention of severe consequences of the disease (Alzahrani et al. 2020; Pocsfalvi et al. 2020).

MSC-EVs emulate the immunoregulatory characteristics and the regenerative function of MSCs (Fig. 2) (Wiklander et al. 2019). Several studies have shown that intravenous or intratracheal administration of MSC-exosome (Exos) attenuates acute lung injury through decreased levels of inflammatory mediators as well as monocyte infiltration into the lung and subsequently protects alveolar epithelia during acute respiratory distress syndrome (ARDS) in the patients with COVID-19 (Tsuchiya et al. 2020; Rezakhani et al. 2020). MSC-Exos suppress cytokine storm via inhibiting the proinflammatory factors IL- $1 \beta$, IL-6, IL-17, TNF, and IFN- $\gamma$, and promote $\mathrm{M} 2$ phenotype for releasing anti-inflammatory factors, such as IL-4, IL-10, and TGF- $\beta$ (Jayaramayya et al. 2020). These protective effects of MSC-Exos may be linked to exosomal active mitochondria, which promote the switching of macrophage polarization from M1 to M2 state via the enhancement of oxidative phosphorylation (Al-Khawaga and Abdelalim 2020).

Evidence has emphasized that MSCs-EVs exert a considerable role in neuroinflammation, neurogenesis, and neurogenic niches, and thus can be considered as a promising therapeutic strategy in neurological diseases such as stroke. Indeed, MSCs-EVs reduce neuroinflammation through modulating proinflammatory responses and induce neurogenesis 


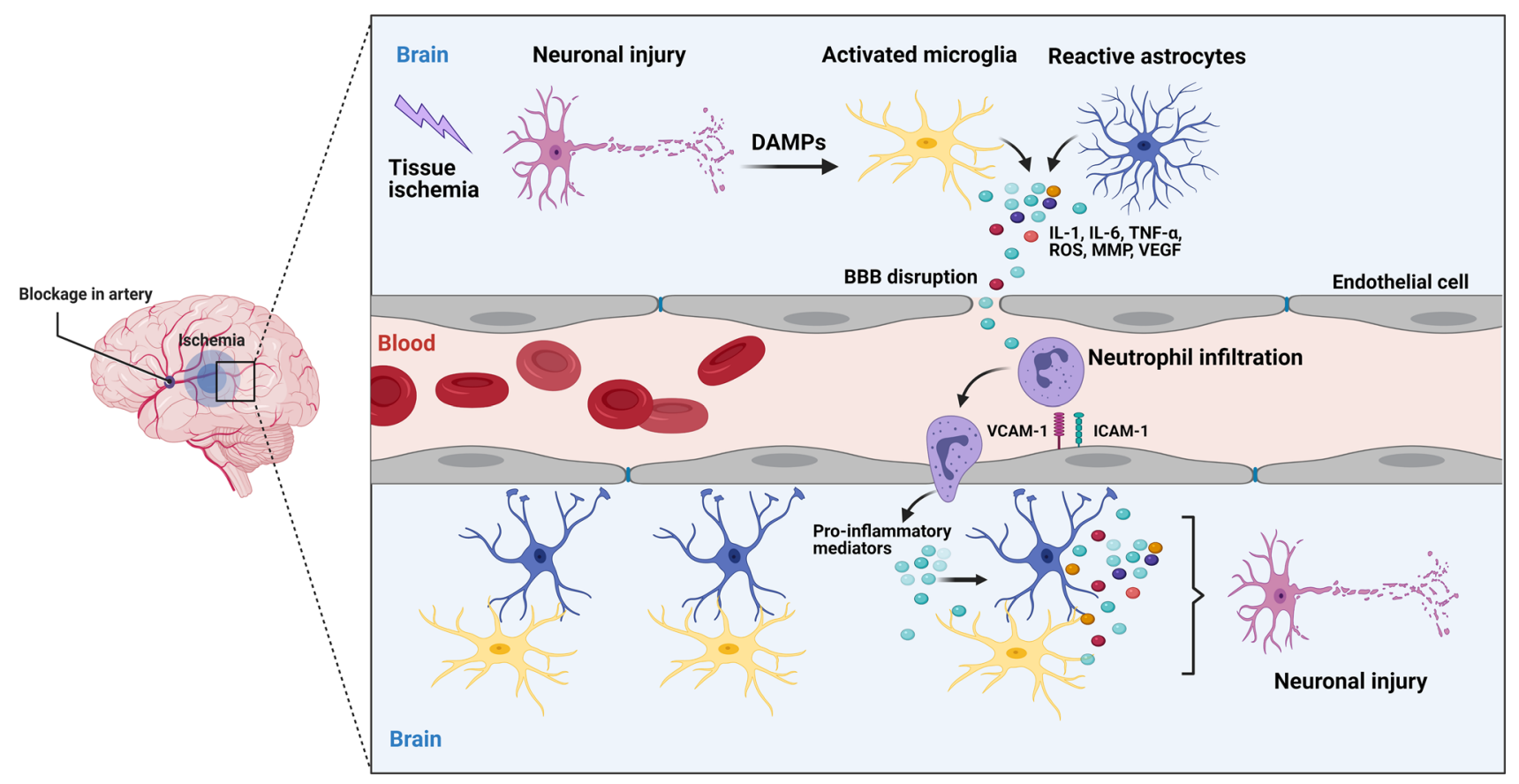

Fig. 2 Formation and composition of extracellular vesicles (EVs) and their therapeutic effects in reducing brain inflammation. A EVs are routinely categorized based upon their biogenesis, size, surface markers, contents and functions, and include exosomes, microvesicles, and apoptotic bodies. Exosomes are produced in the endosomal compartment by inward budding of plasma membrane and formation of early endosomes. Subsequently, early endosomes mature into late endosomal multivesicular bodies (MVBs) which contain membrane-bound intraluminal vesicles. Then intraluminal vesicles, i.e., exosomes release into the extracellular milieu upon fusion of MVBs with the cell's plasma membrane through exocytosis. Microvesicles arise as a

and angiogenesis (Yang et al. 2017), subsequently promoting functional recovery (Table 1). Recently, the results of a systematic review showed that the beneficial effects of MSC-Exos therapy in animal models of stroke were linked to inhibition of inflammation and oxidative stress as well as enhanced angiogenesis, neurogenesis, and neurite remodeling (Dehghani et al. 2020). Pathipati et al. stated that exosomes derived from bone marrow MSCs (BMSC-Exos) are taken up by microglial cells isolated from the injured brain regions of neonatal rats following IS and subsequently glia activation and inflammatory responses are suppressed (2019). One of the most important mechanisms involved in the therapeutic properties of MSC-EVs applied in brain ischemia is the transportation of miRNAs between cells. MSC-EVs contain miRNAs that contribute to neurological functions in animal models of IS through regulation of posttranscriptional gene expression in target cells (Dabrowska et al. 2019a, b).

Moreover, MSC-EVs induce endogenous brain cells to release exosomal miRNAs and improve brain plasticity following IS (Chen and Chopp 2018). Huang et al., for result of direct outward budding of the plasma membrane into extracellular space (blebbing from living cells), and apoptotic bodies are only formed by cells undergoing apoptosis (blebbing from apoptotic cells). B Different EVs contain various markers and cargoes including proteins, lipids, and nucleic acids. C MSC-EVs, including Exosomes and microvesicles, attenuate neuroinflammation mainly through suppressing the activation of astrocytes and microglia and shifting to an anti-inflammatory state. Moreover, MSC-EVs promote angiogenesis and neurite remodeling as well as neurogenesis possibly by inducing the neurogenic niche

instance, have demonstrated that the exosomal miR-124-3p derived from microglia stimulates their polarity towards an anti-inflammatory state while inhibiting proinflammatory responses, eventually trigging the repair of damaged neurons. This evidence suggests that upregulating miRNAs such as miR-124-3p in MSC-EV s could be considered as a therapeutic approach for targeting neuroinflammation and recovering the damaged neurons following stroke (2018). In another study, adipose MSC-derived exosomes (ADMSCExos) enriched with miR-30d-5p have displayed the protective effects in patients with IS as well as in animal models of stroke. Exosomes derived from microRNA-30d-5p-overexpressing ADMSC mostly attenuated brain injury during the acute phase of stroke by suppressing autophagy and stimulating M2 microglial polarization (Jiang et al. 2018). Moreover, Deng et al. have reported that BMSCs deliver miR-138-5p to the astrocytes by exosomes and remarkably suppress the injury volume and inflammation, presumably via the regulation of the expression of the neutrophil gelatinase-associated lipocalin (LCN2) in an IS mouse model (2019). 
Table 1 The efficacies of MSC-derived EVs on neuroinflammation

\begin{tabular}{|c|c|c|c|c|}
\hline Source of exosome & Disease model & Clinical value & Bioactive components & Ref \\
\hline BMSC & Rat model of TBI & $\begin{array}{l}\text { BMSC-Exos increased brain angiogen- } \\
\text { esis and neurogenesis, and reduced } \\
\text { neuroinflammation }\end{array}$ & NA & (Zhang et al. 2015) \\
\hline BMSC & Rat model of TBI & $\begin{array}{l}\text { BMSC-Exos did not reduce lesion size } \\
\text { but remarkably improved spatial learn- } \\
\text { ing, sensorimotor functional recovery. } \\
\text { Exosome treatment increased neuro- } \\
\text { genesis, and reduced neuroinflamma- } \\
\text { tion. BCMSC-Exos cultured in 3D } \\
\text { scaffolds exerted better outcome in spa- } \\
\text { tial learning compared with BCMSC- } \\
\text { Exos cultured in the 2D condition }\end{array}$ & NA & (Zhang et al. 2017) \\
\hline BMSC & Mouse model of TBI & $\begin{array}{l}\text { Administration of the BMSC-EVs } \\
\text { decreased the levels of proinflamma- } \\
\text { tory cytokine IL-1 } \beta \text { in a dose-depend- } \\
\text { ent manner and suppressed neuroin- } \\
\text { flammation after TBI }\end{array}$ & NA & (Kim et al. 2016) \\
\hline ADMSC & Patient and rat model of IS & $\begin{array}{l}\text { microRNA-30d-5p-overexpressing } \\
\text { ADMSC attenuated brain injury during } \\
\text { the acute phase of stroke by suppress- } \\
\text { ing autophagy and stimulating M } 2 \\
\text { microglial polarization }\end{array}$ & microRNA-30d-5p & (Jiang et al. 2018) \\
\hline BMSC & Mouse model of IS & $\begin{array}{l}\text { microRNA-138-5p-overexpressing } \\
\text { BMSC suppressed the injury volume } \\
\text { and neuroinflammation presumably } \\
\text { via the regulation of the lipocalin } 2 \\
\text { (LCN2) expression }\end{array}$ & microRNA-138-5p & (Deng et al. 2019) \\
\hline UCMSC & Rat model of IS & $\begin{array}{l}\text { UCMSC-Exos and specially exosomes } \\
\text { derived from CCR2-overexpressing } \\
\text { UCMSCs exhibited beneficial effects } \\
\text { on oligodendrogenesis, remyelina- } \\
\text { tion, and polarization, and improved } \\
\text { cognitive functions following stroke. } \\
\text { Exos }{ }^{\mathrm{CCR} 2} \text { inhibited the activation of } \\
\text { macrophages and suppressed M1 } \\
\text { polarization of microglia }\end{array}$ & CCR2 & (Yang et al. 2020) \\
\hline BMSC & Rat model of IS & $\begin{array}{l}\text { BMSC-Exos in combination with } \\
\text { rosuvastatin improved the functional } \\
\text { recovery, promoted neuroprotection, } \\
\text { and reduced neuroinflammation and } \\
\text { cell death }\end{array}$ & NA & (Safakheil 2020) \\
\hline ADMSC & Rat model of IS & $\begin{array}{l}\text { Exosomes derived from miRNA-126- } \\
\text { overexpressing ADMSCs promoted } \\
\text { functional recovery by improving } \\
\text { neurogenesis and suppressing neuroin- } \\
\text { flammation }\end{array}$ & miRNA-126 & (Geng et al. 2019) \\
\hline ADMSC & Rat model of IS & $\begin{array}{l}\text { The combination of ADMSCs and } \\
\text { ADMSC-Exos significantly suppressed } \\
\text { production of ROS and oxidative stress } \\
\text { and subsequently inflammation in the } \\
\text { setting of ischemia-reperfusion injury } \\
\text { in AIS animals }\end{array}$ & NA & (Chen et al. 2016) \\
\hline BMSC & Mouse model of ALS & $\begin{array}{l}\text { BMSC-EVs primed with IFN- } \gamma \text { sup- } \\
\text { pressed neuroinflammation via specific } \\
\text { immunomodulatory miRNAs acting on } \\
\text { microglia more effective than unprimed } \\
\text { BMSCs }\end{array}$ & $\begin{array}{l}\text { miR-467f } \\
\text { miR-466q }\end{array}$ & (Giunti et al. 2021) \\
\hline
\end{tabular}


Table 1 (continued)

\begin{tabular}{|c|c|c|c|c|}
\hline Source of exosome & Disease model & Clinical value & Bioactive components & Ref \\
\hline BMSC & Mouse model of SCI & $\begin{array}{l}\text { BMSC-EVs under hypoxic precondi- } \\
\text { tioning promoted functional recovery } \\
\text { and suppressed neuroinflammation } \\
\text { following spinal cord injury by shifting } \\
\text { microglial M1/M2 polarization }\end{array}$ & miR-216a-5p & (Liu et al. 2020a, b) \\
\hline WJMSC & Rat model of perinatal brain injury & $\begin{array}{l}\text { Intranasally administration of WJMSC- } \\
\text { EXos reduced microglia-mediated neu- } \\
\text { roinflammation in rats with perinatal } \\
\text { brain injury }\end{array}$ & NA & (Thomi et al. 2019) \\
\hline
\end{tabular}

$B M S C$ bone marrow MSC, ADMSC adipose MSC, UCMSC umbilical cord MSC; WJMSC Wharton's jelly MSC, AIS acute ischemic stroke, ALS amyotrophic lateral sclerosis, $S C I$ spinal cord injury, $N A$ not available

Recently, researchers have evaluated the effects of exosomes derived from umbilical cord MSCs (UCMSCExos) and also exosomes derived from CCR2-overexpressing UCMSCs on post-stroke cognitive impairment. Both naive and enriched UCMSC-Exos exhibited beneficial effects on oligodendrogenesis, remyelination, and polarization, and improved cognitive functions following stroke. However, exosomes enriched by CCR2 (Exos ${ }^{\mathrm{CCR} 2}$ ) showed remarkable efficiency compared to naive exosomes (Yang et al. 2020). CCL2 is highly expressed in the injured tissues and triggers the activation and recruitment of resident microglia in the ischemic injury site (Tian et al. 2017; $\mathrm{Li}$ et al. 2020). Moreover, CCL2 promotes infiltration of CCR2-expressing cells, including peripheral monocytes, $\mathrm{T}$ lymphocytes, and natural killer cells, to the injured parenchyma (Semple et al. 2010). Exos ${ }^{\mathrm{CCR} 2}$ through binding to CCL2 inhibited the infiltration and activation of macrophages and suppressed M1 polarization of microglia/ macrophages (Yang et al. 2020).

Evidence indicated that the delivery route of MSC-EVs can also impact their therapeutic efficiency. In contrast to MSCs, intravenously administration of EVs cannot reduce stroke-induced neuroinflammation. However, intra-arterially injection of BMSC-EVs alleviates ischemia-induced glial cell activation and infiltration of $\mathrm{T}$ cytotoxic lymphocytes and significantly decreases the proinflammatory cytokines and chemokines in a rat model of IS (Dabrowska et al., 2019a, b). Since stroke is a common and devastating complication during SARS-CoV-2 infection and given the promising results of using stem cells or their secretomes in early studies as well as confirmed positive effects of using MSC-EVs for reducing neuroinflammation in animal models of stroke, MSC-EVs can apply at least as the supportive treatment in COVID-19 patients for targeting primary or secondary neuroinflammation induced by direct CNS infection or cytokine storm, respectively (Sriwastava et al. 2021; Amruta et al. 2021; Dabrowska et al. 2021).

\section{Conclusion and Future Prospects}

MSC-EVs are emerging therapeutics for a wide range of inflammatory and degenerative disorders, and are at the beginning of the path to translate from preclinical studies into clinical trials (Forsberg et al. 2020). MSC-EVs possess many advantages compared to classical cell therapy. Higher immunomodulatory effects of MSC-EVs with low immunogenicity, the ability to scalable production and storage of $\mathrm{EVs}$ for further usage, make them as promising therapeutic tool to treat neurodegenerative disorders, such as COVID-19-inducedstroke, in which neuroinflammation plays a determining role in the patient's fate (Schultz et al. 2021; Giunti et al. 2021).

It is claimed that MSC-EVs exert superior rehabilitation effects than MSC therapy in stroke conditions which may be due to the higher capacity of EVs to cross the BBB and thus reach ischemic regions faster compared with MSCs (Moon et al. 2019). Most preclinical studies indicated that either naive or engineered MSC-EVs exert influential therapeutic effects in stroke and neurological injury (Zhang et al. 2019). The results of these studies have demonstrated the impact of MSC-EVs in improving functional recovery, increased neurogenesis as well as reduced neuroinflammation. Although, MSC-EVs can act as anti-inflammatory agents to modulate inflammatory conditions, however, another advantage of MSC-EVs is the possibility to payload with other therapeutic agents (O'Driscoll 2020). For instance, in terms of the COVID- 19, antiviral-loaded EVs can be administrated intranasally or by inhalation to target the nasal mucosa and the lungs, as two sites where the coronavirus is frequently localized (Popowski et al. 2021). Moreover, EVs have the potential to be modified by exerting molecular engineering approaches to optimize desired functions. For example, genetically modified MSC-EVs enriched by miRNA-124 are assessed in acute IS patients in phase 1-2 clinical trials (Öztürk et al. 2021). 
However, the clinical use of MSC-EVs is still debatable due to the complexity of MSCs given the tissue origin and also cell culture conditions (Wiklander et al. 2019). In addition, EVs heterogeneity, isolation protocols and subsequently yield and purity, detection and characterization methods, delivery routes, effective dosages, application times, and end-points have remained a controversial challenge. Therefore, biomanufacturing standards and standardized criteria need to be considered for the therapeutic application of MSC-EVs, particularly to treat severe and inflammatory conditions such as COVID-19-induced stroke (Forsberg et al. 2020). For instance, since the sustained inflammatory response and hypercoagulability are considered as the hallmark or consequence of infection with SARS-CoV-2, it is proposed that procoagulant factors and proinflammatory cytokines present in the EVs cargo must be removed before administration (Lee et al. 2021). Moreover, estimating the optimal efficient dose of MSC-EVs is one of the criteria which must be considered. Recently, Otero-Ortega et al. evaluated various doses of EVs including 50, 100, or $200 \mu \mathrm{g}$ intravenously in rat models of stroke. They reported $50 \mu \mathrm{g}$ of MSC-EVs can effectively promote brain protection, repair, and recovery following a subcortical IS. Nonetheless, the dosages lower than $50 \mu \mathrm{g}$ of EVs were not sufficient and effective (2020).

Despite the favorite outcomes in small animal models, well-established non-human primate models are required to evaluate the safety and efficacy of MSC-EVs in nonCOVID and COVID-19-induced stroke. Furthermore, well-controlled and rationally designed clinical studies using naïve MSC-EVs derived from different tissue as well as genetically engineered MSC-EVs are needed to investigate their efficiency and adverse effects.

Author Contributions SS and AG designed the study. LNB wrote the manuscript. FZ, AAK and MSS collected the data. All authors read the final version and revised it.

Funding Not applicable.

Data Availability The authors confirm that the data supporting the findings of this study are available within the article.

\section{Declarations}

Conflict of interest The authors declare that there is no conflict of interest.

Ethical Approval This article does not contain any studies with human participants or animals performed by any of the authors.

Informed Consent This article does not contain any studies with human participants so it is not applicable.

Consent to Participate Not applicable.
Consent to Publish Not applicable.

\section{References}

Alam SB, Willows S, Kulka M, Sandhu JK (2020) Severe acute respiratory syndrome coronavirus 2 may be an underappreciated pathogen of the central nervous system. Eur J Neurol 27:2348-2360. https://doi.org/10.1111/ene.14442

Al-Khawaga S, Abdelalim EM (2020) Potential application of mesenchymal stem cells and their exosomes in lung injury: an emerging therapeutic option for COVID-19 patients. Stem Cell Res Ther 11:437. https://doi.org/10.1186/s13287-020-01963-6

Almeida PGC, Nani JV, Oses JP, Brietzke E, Hayashi MAF (2020) Neuroinflammation and glial cell activation in mental disorders Priscila. Brain Behav Immun Heal 2:100034

Alzahrani FA, Saadeldin IM, Ahmad A et al (2020) The Potential use of mesenchymal stem cells and their derived exosomes as immunomodulatory agents for COVID-19 patients. Stem Cells Int. https://doi.org/10.1155/2020/8835986

Amruta N, Chastain WH, Paz M et al (2021) SARS-CoV-2 mediated neuroinflammation and the impact of COVID-19 in neurological disorders. Cytokine Growth Factor Rev 58:1-15

Berger JR (2020) COVID-19 and the nervous system. J Neurovirol 26:143-148

Bernaus A, Blanco S, Sevilla A (2020) Glia crosstalk in neuroinflammatory diseases. Front Cell Neurosci 14:209. https://doi.org/10. 3389/fncel.2020.00209

Bodro M, Compta Y, Sánchez-Valle R (2021) Presentations and mechanisms of CNS disorders related to COVID-19. Neurol Neuroimmunol Neuroinflammat 8:e923

Bronzuoli MR, Iacomino A, Steardo L, Scuderi C (2016) Targeting neuroinflammation in Alzheimer's disease. J Inflamm Res 9:199-208

Campbell BCV, Khatri P (2020) Stroke. The Lancet 396:129-142. https://doi.org/10.1016/s0140-6736(20)31179-x

Cekanaviciute E, Buckwalter MS (2016) Astrocytes: integrative regulators of neuroinflammation in stroke and other neurological diseases. Neurotherapeutics 13:685-701

Chen J, Chopp M (2018) Exosome therapy for stroke. Stroke 49:1083-1090

Chen KH, Chen CH, Wallace CG et al (2016) Intravenous administration of xenogenic adipose-derived mesenchymal stem cells (ADMSC) and ADMSC-derived exosomes markedly reduced brain infarct volume and preserved neurological function in rat after acute ischemic stroke. Oncotarget 7:74537-74556

Chigr F, Merzouki M, Najimi M (2020) Autonomic brain centers and pathophysiology of COVID-19. ACS Chem Neurosci 11:15201522. https://doi.org/10.1021/acschemneuro.0c00265

Colombo E, Farina C (2016) Astrocytes: key regulators of neuroinflammation. Trends Immunol 37:608-620. https://doi.org/10.1016/j. it.2016.06.006

Dabrowska S, Andrzejewska A, Lukomska B, Janowski M (2019a) Neuroinflammation as a target for treatment of stroke using mesenchymal stem cells and extracellular vesicles. J Neuroinflammation 16:178

Dabrowska S, Andrzejewska A, Strzemecki D, Muraca M, Janowski M, Lukomska B (2019b) Human bone marrow mesenchymal stem cell-derived extracellular vesicles attenuate neuroinflammation evoked by focal brain injury in rats. J Neuroinflammat 16:216

Dabrowska S, Andrzejewska A, Janowski M, Lukomska B (2021) Immunomodulatory and regenerative effects of mesenchymal stem cells and extracellular vesicles: therapeutic outlook 
for inflammatory and degenerative diseases. Front Immunol 11:591065

De Melo GD, Lazarini F, Levallois S et al (2020) COVID-19-associated olfactory dysfunction reveals SARS-CoV-2 neuroinvasion and persistence in the olfactory system. bioRxiv. https://doi.org/ 10.1101/2020.11.18.388819

Dehghani L, Hashemi SM, Saadatnia M et al (2020) Stem cellderived exosomes as treatment for stroke: a systematic review. Stem Cell Rev Rep 17:428-438. https://doi.org/10.1007/ s12015-020-10024-7

Deng Y, Chen D, Gao F et al (2019) Exosomes derived from microRNA-138-5p- overexpressing bone marrow-derived mesenchymal stem cells confer neuroprotection to astrocytes following ischemic stroke via inhibition of LCN2. J Biol Eng 13:71

Ellul M, Benjamin L, Singh B et al (2020) Neurological associations of COVID-19. Lancet 19:767-783. https://doi.org/10.2139/ssrn. 3589350

Ferro JM, Infante J (2021) Cerebrovascular manifestations in hematological diseases: an update. J Neurol. https://doi.org/10.1007/ s00415-021-10441-9

Fifi JT, Mocco J (2020) COVID-19 related stroke in young individuals. Lancet Neurol 19:713-715

Finsterer J, Stollberger C (2020) Update on the neurology of COVID19. J Med Virol 92:2316-2318. https://doi.org/10.1002/jmv. 26000

Forsberg MH, Kink JA, Hematti P, Capitini CM (2020) Mesenchymal stromal cells and exosomes: progress and challenges. Front Cell Dev Biol 8:665. https://doi.org/10.3389/fcell.2020.00665

Geng W, Tang H, Luo S et al (2019) Exosomes from mirna-126-modified adscs promotes functional recovery after stroke in rats by improving neurogenesis and suppressing microglia activation. Am J Transl Res 11:780-792

Giunti D, Marini C, Parodi B et al (2021) Role of miRNAs shuttled by mesenchymal stem cell-derived small extracellular vesicles in modulating neuroinflammation. Sci Rep 11:1740

Gorji A, Khaleghi Ghadiri M (2021) Potential roles of micronutrient deficiency and immune system dysfunction in the coronavirus disease 2019 (COVID-19) pandemic. Nutrition 82:111047

He J, Zhu G, Wang G, Zhang F (2020) Oxidative stress and neuroinflammation potentiate each other to promote progression of dopamine neurodegeneration. Oxid Med Cell Longev 2020:6137521

Huang S, Ge X, Yu J et al (2018) Increased miR-124-3p in microglial exosomes following traumatic brain injury inhibits neuronal inflammation and contributes to neurite outgrowth via their transfer into neurons. FASEB J 32:512-528

Jarrahi A, Ahluwalia M, Khodadadi H et al (2020) Neurological consequences of COVID-19: what have we learned and where do we go from here? J Neuroinflammation 17:286. https://doi.org/10. 1186/s12974-020-01957-4

Jayaraj RL, Azimullah S, Beiram R, Jalal FY, Rosenberg GA (2019) Neuroinflammation: friend and foe for ischemic stroke. J Neuroinflammation 16:142

Jayaramayya K, Mahalaxmi I, Subramaniam MD et al (2020) Immunomodulatory effect of mesenchymal stem cells and mesenchymal stem-cell-derived exosomes for COVID-19 treatment. BMB Rep 53:400-412. https://doi.org/10.5483/BMBRep.2020.53.8.121

Jiang M, Wang H, Jin M et al (2018) Exosomes from MiR-30d-5pADSCs reverse acute ischemic stroke-induced, autophagy-mediated brain injury by promoting M2 microglial/macrophage polarization. Cell Physiol Biochem 47:864-878

Jiang CT, Wu WF, Deng YH, Ge JW (2020) Modulators of microglia activation and polarization in ischemic stroke (Review). Mol Med Rep 21:2006-2018

Jurga AM, Paleczna M, Kuter KZ (2020) Overview of general and discriminating markers of differential microglia phenotypes.
Front Cell Neurosci 14:198. https://doi.org/10.3389/fncel.2020. 00198

Kim DK, Nishida H, An SY et al (2016) Chromatographically isolated CD63+CD81+ extracellular vesicles from mesenchymal stromal cells rescue cognitive impairments after TBI. Proc Natl Acad Sci USA 113:170-175

Lanza M, Casili G, Campolo M et al (2021) Immunomodulatory effect of microglia-released cytokines in gliomas. Brain Sci 11:466

Lee B-C, Kang I, Yu K-R (2021) Therapeutic features and updated clinical trials of mesenchymal stem cell (MSC)-derived exosomes. J Clin Med 10:711. https://doi.org/10.3390/jcm10040711

Leisman DE, Ronner L, Pinotti R et al (2020) Cytokine elevation in severe and critical COVID-19: a rapid systematic review, metaanalysis, and comparison with other inflammatory syndromes. Lancet Respir 8:1233-1244

Li L, Lou W, Li H, Zhu Y, Huang X (2020) Up-regulated CCL2 promotes ischemic stroke via chemokine signaling pathway. Ann Vasc Surg 68:476-486

Lian L, Zhang Y, Liu L et al (2021) Neuroinflammation in ischemic stroke: focus on MicroRNA-mediated polarization of microglia. Front Mol Neurosci 13:612439

Liu R, Pan M-X, Tang J-C et al (2017) Role of neuroinflammation in ischemic stroke. Neuroimmunol Neuroinflammat 4:158

Liu CY, Wang X, Liu C, Zhang HL (2019) Pharmacological targeting of microglial activation: new therapeutic approach. Front Cell Neurosci 13:514. https://doi.org/10.3389/fncel.2019.00514

Liu LR, Liu JC, Bao JS et al (2020a) Interaction of microglia and astrocytes in the neurovascular unit. Front Immunol 11:1024

Liu W, Rong Y, Wang J et al (2020b) Exosome-shuttled miR-216a-5p from hypoxic preconditioned mesenchymal stem cells repair traumatic spinal cord injury by shifting microglial M1/M2 polarization. J Neuroinflammation 17:47

Mendiola AS, Cardona AE (2018) The IL-1 $\beta$ phenomena in neuroinflammatory diseases. J Neural Transm 125:781-795

Merkler AE, Parikh NS, Mir S et al (2020) Risk of ischemic stroke in patients with coronavirus disease 2019 (COVID-19) vs patients with influenza. Jamaneurology 77:1366-1372

Moon GJ, Sung JH, Kim DH et al (2019) Application of mesenchymal stem cell-derived extracellular vesicles for stroke: biodistribution and MicroRNA study. Transl Stroke Res 10:509-521

Murta V, Villarreal A, Ramos AJ (2020) Severe acute respiratory syndrome coronavirus 2 impact on the central nervous system: are astrocytes and microglia main players or merely bystanders? ASN Neuro 12:1-17. https://doi.org/10.1177/1759091420954960

Nannoni S, de Groot R, Bell S, Markus HS (2021) Stroke in COVID-19: a systematic review and meta-analysis. Int J Stroke 16:137-149

Ntaios G, Michel P, Georgiopoulos G et al (2020) Characteristics and outcomes in patients with COVID-19 and acute ischemic stroke. Stroke 51:e254-e258

O’Driscoll L (2020) Extracellular vesicles from mesenchymal stem cells as a Covid-19 treatment. Drug Discov Today 25:1124-1125. https://doi.org/10.1016/j.drudis.2020.04.022

Otero-Ortega L, Laso-García F, Gómez-de Frutos MC et al (2020) Low dose of extracellular vesicles identified that promote recovery after ischemic stroke. Stem Cell Res Ther 11:70. https://doi.org/ 10.1186/s13287-020-01601-1

Öztürk S, Elçin AE, Koca A, Elçin YM (2021) Therapeutic applications of stem cells and extracellular vesicles in emergency care: futuristic perspectives. Stem Cell Rev Rep 2:390-410. https://doi. org/10.1007/s12015-020-10029-2

Pathipati P, LeCuyer M, Faustino J, Strivelli J, Phinney DG, Vexler Z (2019) Interaction of mesenchymal stem cell (MSC)-derived exosomes with microglia isolated after acute neonatal stroke. J Cereb Blood Flow Metab 39:364

Pezzini A, Padovani A (2020) Lifting the mask on neurological manifestations of COVID-19. Nat Rev Nephrol 16:636-644 
Pocsfalvi G, Mammadova R, Ramos Juarez AP et al (2020) COVID-19 and extracellular vesicles: an intriguing interplay. Kidney Blood Press Res 45:661-670. https://doi.org/10.1159/000511402

Popowski KD, Dinh PC, George A et al (2021) Exosome therapeutics for COVID-19 and respiratory viruses. View. https://doi.org/10. 1002/viw.20200186

Rajkovic O, Pinteaux GP, Pinteaux E (2018) Regenerative medicine therapies for targeting neuroinflammation after stroke. Front Neurol 9:734

Rezaeitalab F, Jamehdar SA, Sepehrinezhad A et al (2021) Detection of SARS-coronavirus- 2 in the central nervous system of patients with severe acute respiratory syndrome and seizures Fariborz. J Neurovirol 2:348-353

Rezakhani L, Fatahian A, Soleimanizadeh A, Rahmati S (2020) Mesenchymal stem cell (MSC)-derived exosomes as a cell-free therapy for patients infected with COVID-19: Real opportunities and range of promises. Chem Phys Lipids 234:105009

Safakheil M, Safakheil H (2020) The effect of exosomes derived from bone marrow stem cells in combination with rosuvastatin on functional recovery and neuroprotection in rats after ischemic stroke. J Mol Neurosci 70:724-737

Schultz IC, Bertoni APS, Wink MR (2021) Mesenchymal stem cellderived extracellular vesicles carrying miRNA as a potential multi target therapy to COVID-19: an in silico analysis. Stem Cell Rev Rep 17:341-356. https://doi.org/10.1007/s12015-021-10122-0

Semple BD, Kossmann T, Morganti-Kossmann MC (2010) Role of chemokines in CNS health and pathology: a focus on the CCL2/ CCR2 and CXCL8/CXCR2 networks. J Cereb Blood Flow Metab 30:459-473

Shah A, Ahmed Shaikh N, Alattar K, Thomas LM, Sabahat U (2020) Stroke as a presenting feature of COVID-19. Int J Clin Med 11:743-749

Skaper SD, Giusti P, Facci L (2017) Microglia and mast cells: two tracks on the road to neuroinflammation. FASEB J 26:3103-3117

Soltani Zangbar H, Gorji A, Ghadiri T (2021) A review on the neurological manifestations of COVID-19 infection: a mechanistic view. Mol Neurobiol 58:536-549

Song E, Zhang C, Israelow B et al (2021) Neuroinvasion of SARSCoV-2 in human and mouse brain. J Exp Med 218:e20202135

Spence JD, De Freitas GR, Pettigrew LC et al (2020) Mechanisms of stroke in COVID-19. Cerebrovasc Dis 49:451-458

Sriwastava S, Tandon M, Podury S et al (2021) COVID-19 and neuroinflammation: a literature review of relevant neuroimaging and CSF markers in central nervous system inflammatory disorders from SARS-COV2. J Neurol. https://doi.org/10.1007/ s00415-021-10611-9

Stephenson J, Nutma E, van der Valk P, Amor S (2018) Inflammation in CNS neurodegenerative diseases. Immunology 154:204-219. https://doi.org/10.1111/imm.12922

Tadi P, Lui F (2020) Acute Stroke. In: StatPearls [Internet]. Treasure Island (FL): StatPearls Publishing. https://www.ncbi.nlm.nih.gov/ books/NBK535369/

Thomi G, Surbek D, Haesler V et al (2019) Exosomes derived from umbilical cord mesenchymal stem cells reduce microglia-mediated neuroinflammation in perinatal brain injury. Stem Cell Res Ther 10:105

Tian DS, Peng J, Murugan M et al (2017) Chemokine CCL2-CCR2 signaling induces neuronal cell death via STAT3 Activation and IL- $1 \beta$ production after status epilepticus. Neurobiol Dis 37:7878-7892
Tsuchiya A, Takeuchi S, Iwasawa T et al (2020) Therapeutic potential of mesenchymal stem cells and their exosomes in severe novel coronavirus disease 2019 (COVID-19) cases. Inflamm Regen 40:14. https://doi.org/10.1186/s41232-020-00121-y

Virani SS, Alonso A, Aparicio HJ et al (2021) Heart disease and stroke statistics-2021 update a report from the American Heart Association. Circulation 143:e254-e743

Whitney NP, Eidem MT, Peng H, Huang Y, Zheng JC (2009) Inflammation mediates varying effects in neurogenesis: relevance to the pathogenesis of brain injury and neurodegenerative disorders. $\mathrm{J}$ Neurochem 108:1343-1359

Wiklander OPB, Brennan ÁM, Lötvall J et al (2019) Advances in therapeutic applications of extracellular vesicles. Transl Med 11:8521

Woldu M, Minzi O, Engidawork E (2020) Prevalence of cardiometabolic syndrome in HIV-infected persons: a systematic review. J Diabetes Metab Disord 19:1671-1683

Xiong XY, Liu L, Yang QW (2016) Functions and mechanisms of microglia/macrophages in neuroinflammation and neurogenesis after stroke. Prog Neurobiol 142:23-44

Yaghi S, Ishida K, Torres J et al (2020) SARS-coV-2 and stroke in a New York healthcare system. Stroke 51:2002-2011

Yang Y, Ye Y, Su X et al (2017) MSCs-derived exosomes and neuroinflammation, neurogenesis and therapy of traumatic brain injury. Front Cell Neurosci 11:55

Yang HC, Wu R, Zheng HQ et al (2020) C-C chemokine receptor type 2-overexpressing exosomes alleviated experimental post-stroke cognitive impairment by enhancing microglia/macrophage M2 polarization. World J Stem Cells 12:152-167

Zhang H, Jensen K, Yang BZ et al (2015) Effect of exosomes derived from multipluripotent mesenchymal stromal cells on functional recovery and neurovascular plasticity in rats after traumatic brain injury. J Neurosurg 122:856-867

Zhang Y, Chopp M, Zhang ZG et al (2017) Systemic administration of cell-free exosomes generated by human bone marrow derived mesenchymal stem cells cultured under $2 \mathrm{D}$ and $3 \mathrm{D}$ conditions improves functional recovery in rats after traumatic brain injury. Neurochem Int 111:69-81

Zhang ZG, Buller B, Chopp M (2019) Exosomes — beyond stem cells for restorative therapy in stroke and neurological injury. Nat Rev Neurol 15:193-203

Zhang H, Kang Z, Gong H et al (2020) Digestive system is a potential route of COVID-19: an analysis of single-cell coexpression pattern of key proteins in viral entry process. Gut 69:1010-1018. https://doi.org/10.1136/gutjnl-2020-320953

Zhang S, Zhang J, Wang C et al (2021a) COVID-19 and ischemic stroke: mechanisms of hypercoagulability (Review). Int J Mol Med 47:21

Zhang W, Tian T, Gong SX et al (2021b) Microglia-associated neuroinflammation is a potential therapeutic target for ischemic stroke. Neural Regen Res 16:6-11

Zheng ZV, Wong KCG (2019) Microglial activation and polarization after subarachnoid hemorrhage. Neuroimmunol Neuroinflammat $6: 1$

Publisher's Note Springer Nature remains neutral with regard to jurisdictional claims in published maps and institutional affiliations. 